\title{
Analysis of 100Gbps Based Optical AP-DCDM Network
}

\author{
Naina Khanna ${ }^{1}$, AyushJouhari ${ }^{2}$, Dr.SoniChanglani ${ }^{3}$ \\ M.Tech Scholar, ECE Department, LNCTS, Bhopal MP, India ${ }^{1}$ \\ Assistant Professor, ECE Department, LNCTS, Bhopal MP, India ${ }^{2}$ \\ Professor, ECE Department, LNCTS, Bhopal MP, India ${ }^{3}$
}

\begin{abstract}
Multiplexing is a method by which multiple analog message signals or digital data streams are combined into one signal over a shared medium. The aim is to share an expensive resource. TDM is the most widely used multiplexing technique in today's communication, in which the main issue is a clock recovery that may render the system highly complicated and costly for TDM system. Therefore, many investigations have been done to design and develop reliable and cost-effective clock recovery modules for TDM in both electrical and optical versions. These problems can be resolved by an absolute polar duty cycle division multiplexing (APDCDM). APDCDM also decreases the complexity of receiver and improved the capacity of channels as compare to the DCDM system. In this workfive user APDCDM system has been designed and each user has data rate of $20 \mathrm{Gbps}, 20 \times 5=100$ Gbps signal is transmitted through optical fiber cable and data is successfully transmitted up to $75 \mathrm{~km}$ distance using a standard single mode fiber.
\end{abstract}

\section{Keywords: APDCDM, BER.}

\section{INTRODUCTION}

Multiplexing is one of the fundamental necessities in Where, ' $n$ ' represents number of multiplexing users.

today's digital communications. Multiplexing allows For example, assigning the duty cycle value for 5 users multiple users (or data inputs) to share the bandwidth of using DCDM technique:

the transmission medium. In existing systems, the medium is normally shared based on time slot (TDM), carrier For the $1^{\text {st }}$ user $=T_{\mathrm{s}} / 6$.

frequency $(\mathrm{FDM})$ or spectrum coding $(\mathrm{CDM})$. The goals For the $2^{\text {nd }}$ user $=2 \mathrm{~T}_{\mathrm{s}} / 6$.

of all multiplexing techniques are to support as many users For the $3^{\text {rd }}$ user $=3 \mathrm{~T}_{\mathrm{s}} / 6$.

at as high speed and at the lowest cost possible [3].

For the $4^{\text {th }}$ user $=4 \mathrm{~T}_{\mathrm{s}} / 6$.

For the $5^{\text {th }}$ user $=5 \mathrm{~T}_{\mathrm{s}} / 6$.

However, for multiplexing high number of users with high data rates, high speed multiplexer and de-multiplexer are required. At higher speeds clock recovery is another essential issue which limit the conventional multiplexing techniques.

Realizing these problems the design of five users Absolute Polar Duty Cycle Division Multiplexing (APDCDM) based Optical communication system has been proposed in this paper.

The absolute polar duty cycle division multiplexing is a variant of DCDM. APDCDM require less bandwidth and less energy for transmission as compared to DCDM technique.

It is based on having each channel modulated with a unique RZ duty cycle. In this technique each multiplexing user transmits bit ' 0 ' with zero volts and for the case of bit one, the odd users transmit with $+\mathrm{A}$ volts and the even users transmit with $-\mathrm{A}$ volts.

For $n=5,2^{5}=32$ possible combinations are shown in table- 1

Table 1: Possible combination of five user APDCDM System

\begin{tabular}{|c|c|c|c|c|c|c|c|c|c|c|c|c|c|c|c|c|}
\hline Cases & $\mathbf{1}$ & $\mathbf{2}$ & $\mathbf{3}$ & $\mathbf{4}$ & $\mathbf{5}$ & $\mathbf{6}$ & $\mathbf{7}$ & $\mathbf{8}$ & $\mathbf{9}$ & $\mathbf{1 0}$ & $\mathbf{1 1}$ & $\mathbf{1 2}$ & $\mathbf{1 3}$ & $\mathbf{1 4}$ & $\mathbf{1 5}$ & $\mathbf{1 6}$ \\
\hline User-5 & 0 & 0 & 0 & 0 & 0 & 0 & 0 & 0 & 0 & 0 & 0 & 0 & 0 & 0 & 0 & 0 \\
\hline User-4 & 0 & 0 & 0 & 0 & 0 & 0 & 0 & 0 & 1 & 1 & 1 & 1 & 1 & 1 & 1 & 1 \\
\hline User-3 & 0 & 0 & 0 & 0 & 1 & 1 & 1 & 1 & 0 & 0 & 0 & 0 & 1 & 1 & 1 & 1 \\
\hline User-2 & 0 & 0 & 1 & 1 & 0 & 0 & 1 & 1 & 0 & 0 & 1 & 1 & 0 & 0 & 1 & 1 \\
\hline User-1 & 0 & 1 & 0 & 1 & 0 & 1 & 0 & 1 & 0 & 1 & 0 & 1 & 0 & 1 & 0 & 1 \\
\hline
\end{tabular}

\begin{tabular}{|c|r|r|r|r|r|r|r|r|r|r|r|r|r|r|r|r|}
\hline Cases & $\mathbf{1 7}$ & $\mathbf{1 8}$ & $\mathbf{1 9}$ & $\mathbf{2 0}$ & $\mathbf{2 1}$ & $\mathbf{2 2}$ & $\mathbf{2 3}$ & $\mathbf{2 4}$ & $\mathbf{2 5}$ & $\mathbf{2 6}$ & $\mathbf{2 7}$ & $\mathbf{2 8}$ & $\mathbf{2 9}$ & $\mathbf{3 0}$ & $\mathbf{3 1}$ & $\mathbf{3 2}$ \\
\hline User-5 & 1 & 1 & 1 & 1 & 1 & 1 & 1 & 1 & 1 & 1 & 1 & 1 & 1 & 1 & 1 & 1 \\
\hline User-4 & 0 & 0 & 0 & 0 & 0 & 0 & 0 & 0 & 1 & 1 & 1 & 1 & 1 & 1 & 1 & 1 \\
\hline User-3 & 0 & 0 & 0 & 0 & 1 & 1 & 1 & 1 & 0 & 0 & 0 & 0 & 1 & 1 & 1 & 1 \\
\hline User-2 & 0 & 0 & 1 & 1 & 0 & 0 & 1 & 1 & 0 & 0 & 1 & 1 & 0 & 0 & 1 & 1 \\
\hline User-1 & 0 & 1 & 0 & 1 & 0 & 1 & 0 & 1 & 0 & 1 & 0 & 1 & 0 & 1 & 0 & 1 \\
\hline
\end{tabular}

Based on the linear distribution of duty cycle, the $i^{\text {th }}$ Five user APDCDM has 32 cases out of them some cases multiplexing user transmits bit 1 within $T_{\mathrm{i}}$ second which is calculated as:

$T_{i}=\frac{i \times T_{S}}{(n+1)}$ are discuss below as-

Case-4,Symbol-1 generates by User-1 \& User-2 Symbol-0 generates by User-3, User-4\& User-5 


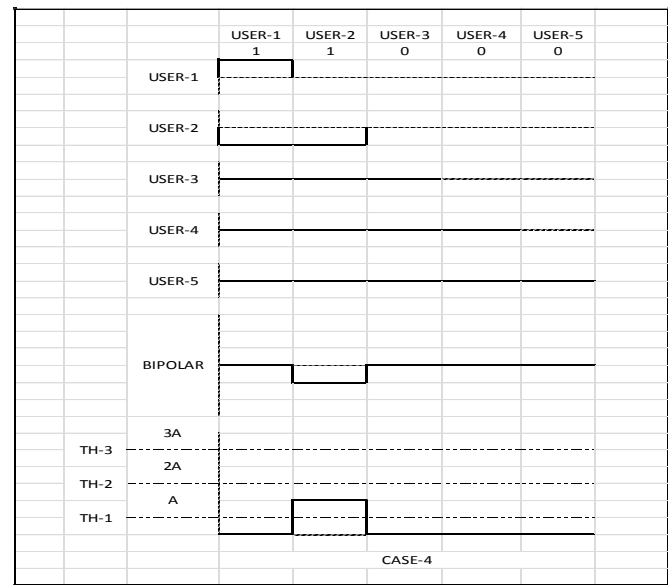

Figure 1.1: Wave form of case-4 for five user APDCDM system

Case-8, Symbol-1 generates by User-1, User-2\&User-3 Symbol-0 generates by User-4 \&User-5

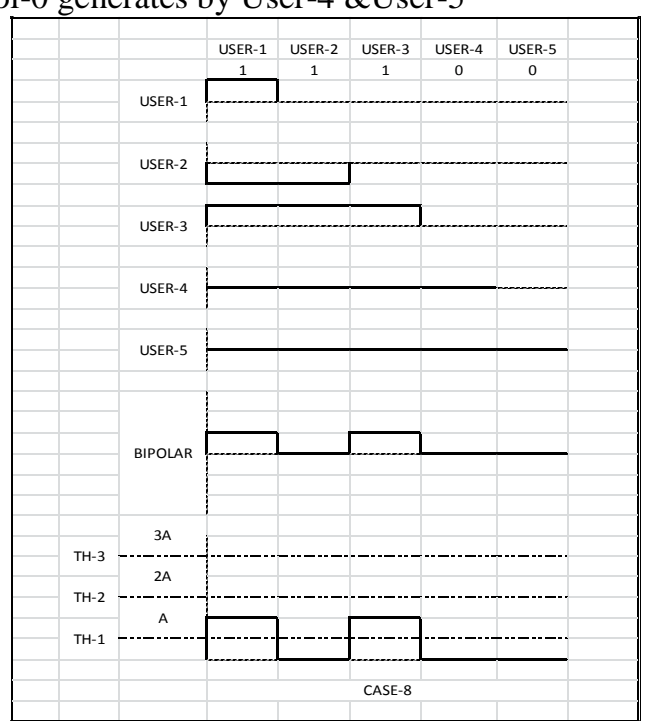

Figure 1.2: Wave form of case- 8 for five user APDCDM System

Case-16, Symbol-1 generates by User-1,2,3 and 4 Symbol-0 generates by User-5

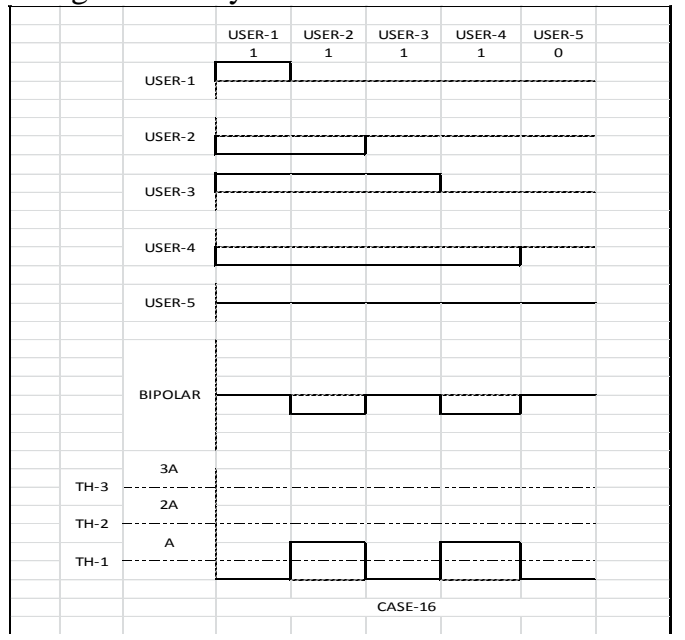

Figure 1.3: Wave form of case-16 for five user APDCDM system

Case-32,Symbol-1 generates by User-1, 2, 3, 4 and 5

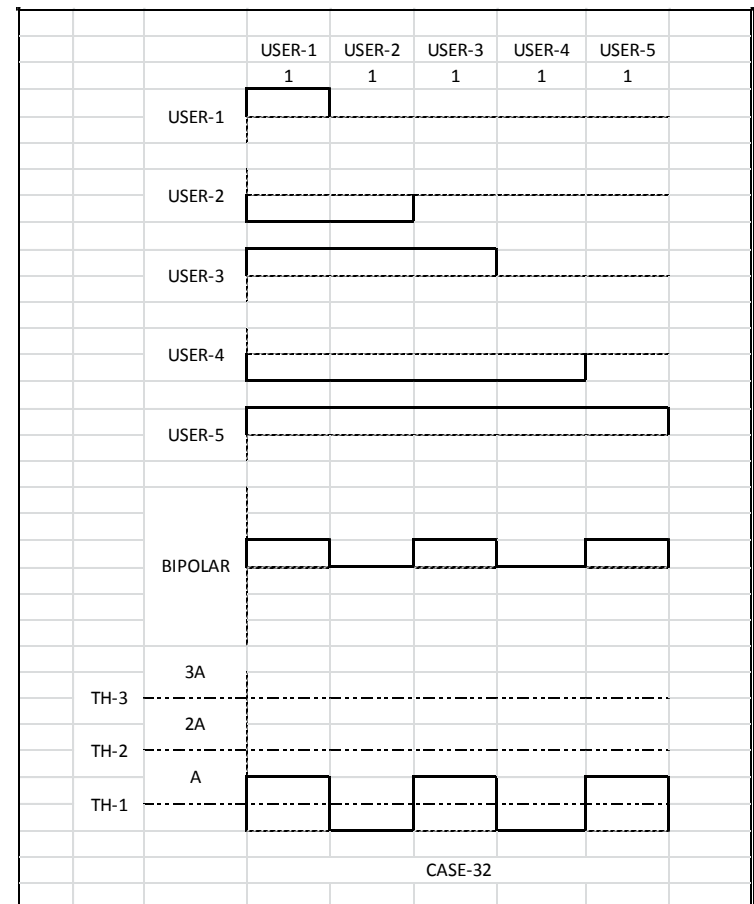

Figure 1.4: Wave form of case-32 for five user APDCDM system

\section{SIMULATION SETUP}

In transmitter section, the five users send their data at different duty cycles. There are five user define Bit Sequence Generators (BSG), the output of BSGs are modulated by Return to Zero (RZ) pulse generators. The RZ pulse generator modulates duty cycle of input signal as shown in figure-2. The RZ-PG1 modulates 15\%, RZ-PG2 modulates $30 \%$, RZ-PG3 modulates $45 \%$, RZ-PG4 modulates $60 \%$, and RZ-PG5 modulates $75 \%$. The last $25 \%$ is used for guard band purpose, to avoid symbol overlapping in communication system.

The output of RZ pulse generators are electrically multiplexed using Electrical adder. The Output of electrical adder is passed through the absolute polar circuit and then multiplexed data is converted in optical signal by modulating the continuous wave (CW) laser (light source) and transferred through an optical fiber.

The optical signal is received and detected by a PIN detector which converts the optical signal in electrical form. Then low pass filter (LPF) is used to eliminate the noise that produced in optical fiber

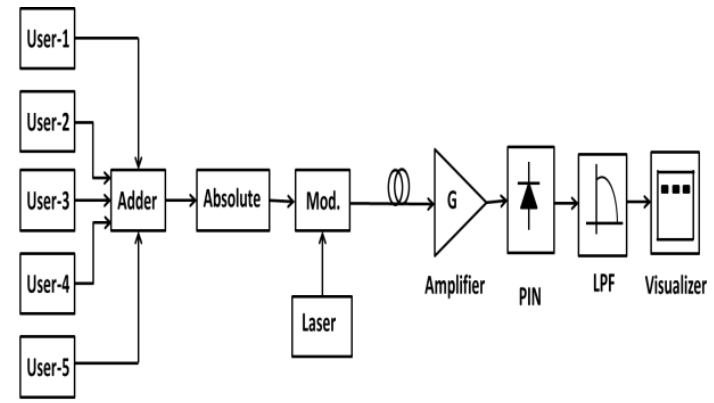

Figure 2: Block diagram of 100 GBPS WDM Optical Network 


\section{RESULTS}

III.I Data Recovery Rules-

The digital receiver uses probability theory for decision making based on that each amplitude has a threshold, below threshold signal is considered as logic-0 otherwise logic-1.There are five users; each user has different data recovery rule for all 32 cases. Here five user uses five sampling points $\left(\mathrm{sp}_{1}, \mathrm{sp}_{2}, \mathrm{sp}_{3}, \mathrm{sp}_{4}\right.$ and $\left.\mathrm{sp}_{5}\right)$ in $\mathrm{x}$-axis (time) and three threshold values $\left(\mathrm{th}_{1}, \mathrm{th}_{2}\right.$ and $\left.\mathrm{th}_{3}\right)$ in $\mathrm{y}$-axis (amplitude).

Table 2: Data recovery rule for User-1

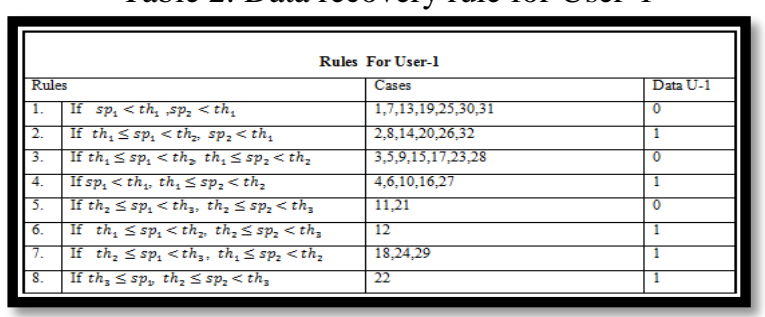

Table 3: Data recovery rule for User-2

\begin{tabular}{|c|c|c|c|}
\hline \multicolumn{4}{|c|}{ Rules For User-2 } \\
\hline \multicolumn{2}{|c|}{ Rules } & Cases & Data U-2 \\
\hline & If $s p_{2}<t h_{1}, s p_{3}<t h_{1}$ & $1,2,13,14,25,26$ & 0 \\
\hline 2. & If $t h_{1} \leq s p_{2}<t h_{2}, s p_{3}<t h_{1}$ & $3,4,15,16,27$ & 1 \\
\hline 3. & If $t h_{1} \leq s p_{2}<t h_{2}, t h_{1} \leq s p_{3}<t h_{2}$ & $5,6,9,10,17,18,28,29$ & 0 \\
\hline 4. & If $s p_{2}<t h_{1}, t h_{1} \leq s p_{3}<t h_{2}$ & $7,8,19,20,30.31 .32$ & 1 \\
\hline 5. & If $t h_{2} \leq s p_{2}<t h_{3}, t h_{1} \leq s p_{3}<t h_{2}$ & 11,12 & 1 \\
\hline 6. & If $t h_{2} \leq s p_{2}<t h_{3}, t h_{2} \leq s p_{3}<t h_{3}$ & 21,22 & 0 \\
\hline 7. & If $t h_{1} \leq s p_{2}<t h_{2}, t h_{2} \leq s p_{3}<t h_{3}$ & 23,24 & 1 \\
\hline
\end{tabular}

Table 4: Data recovery rule for User-3

\begin{tabular}{|c|c|c|c|}
\hline \multicolumn{4}{|c|}{ Rules For User-3 } \\
\hline Rule & & Cases & DataU-3 \\
\hline & If $s p_{3}<t h_{1}, s p_{4}<t h_{1}$ & $1,2,3,4,25,26,27$ & 0 \\
\hline 2. & If $t h_{1} \leq s p_{3}<t h_{2}, s p_{4}<t h_{1}$ & $5,6,7,8,28,29,30,31,32$ & 1 \\
\hline 3. & If $t h_{1} \leq s p_{3}<t h_{2}, t h_{1} \leq s p_{4}<t h_{2}$ & $9,10,11,12,17,18,19,20$ & 0 \\
\hline & If $t h_{2} \leq s p_{3}<t h_{3}, t h_{1} \leq s p_{4}<t h_{2}$ & $21,22,23,24$ & 1 \\
\hline
\end{tabular}

Table 5: Data recovery rule for User-4

\begin{tabular}{|l|l|l|l|}
\hline \multicolumn{4}{|c|}{ Rules For User-4 } \\
\hline Rules & Cases & Data U-4 \\
\hline 1. & If $s p_{4}<t h_{1}, s p_{5}<t h_{1}$ & $1,2,3,4,5,6,7,8$ & 0 \\
\hline 2. & If $t h_{1} \leq s p_{4}<t h_{2}, s p_{5}<t h_{1}$ & $9,10,11,12,13,14,15,16$ & 1 \\
\hline 3. & If $t h_{1} \leq s p_{4}<t h_{2}, t h_{1} \leq s p_{5}<t h_{2}$ & $17,18,19,20,21,22,23,24$ & 0 \\
\hline 4. & If $s p_{4}<t h_{1}, t h_{1} \leq s p_{5}<t h_{2}$ & $25,26,27,28,29,30,31,32$ & 1 \\
\hline
\end{tabular}

Table 6: Data recovery rule for User-5

\begin{tabular}{|c|c|c|}
\hline \multicolumn{3}{|c|}{ Rules For User-5 } \\
\hline Rules & Cases & Data U.5 \\
\hline \begin{tabular}{|l|l|}
1. & If $s p_{5}<t h_{1}$ \\
\end{tabular} & $1,2,3,4,5,6,7,8,9,10,11,12,13,14,15,16$ & 0 \\
\hline \begin{tabular}{|l|l}
2. & If $s p_{5} \geq t h_{1}$ \\
\end{tabular} & 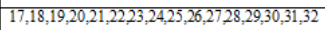 & 1 \\
\hline
\end{tabular}

From above graphs it is conclude that if value at $S p_{i}$ and $S p_{i+1}$ is at same level than $i^{t h}$ user sends zero bit. And if value at $S p_{i}$ is one more level than value at $S p_{i+1}$ than $i^{t h}$ user send one bit. Based on these data recovery rules the multiplexed data of five users is recovered. There are different rules for different of users.

\section{III.II BER versus Length-}

Figure-3, shows BER versus length graph for five user APDCDM system at the data rate of $100 \mathrm{Gbps}$. The multiplexed data is transmitted through a SSMF of $100 \mathrm{~km}$ length; the BER is successfully maintained up to $75 \mathrm{~km}$.

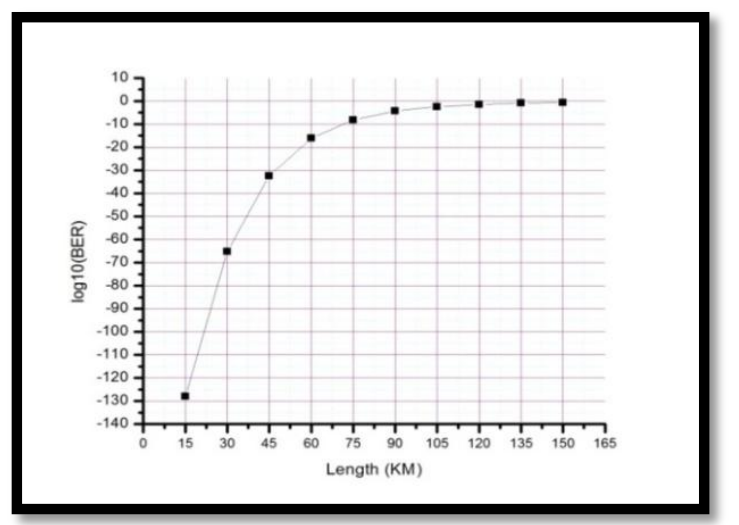

Figure 3: Log BER Vs Length for five user APDCDM

\section{CONCLUSION}

The five user Absolute Polar Duty Cycle Division Multiplexing (APDCDM) based optical communication has been designed successfully. The data recovery rules also designed for five user APDCDM system. The $100 \mathrm{Gbps}$ multiplexed signal is transmitted on the network and data is successfully transmitted up to $75 \mathrm{~km}$ distance.

\section{REFERENCES}

[1]. Amin Malekmohammadi, M.K.Abdullah, A.F.Abas, G.A.Mahdiraji, "Effect of Guard Band on the Performance of APDCDM Technique in $40 \mathrm{~Gb} / \mathrm{s}$ Optical Fibre Communication system". ICP 2010.

[2]. A. Malekmohammadi, M.K. Abdullah, G.A. Mahdiraji,"Analysis of return-to-zero-on-off-keying over absolute polar duty cycle division multiplexing in dispersive transmission medium" IET Optoelectron, Vol. 3, Iss. 4, pp. 197-206., 2009.

[3]. M. K. Abdullah, A. M. Mohammadi, G. A. Mahdiraji, A. F. Abas, and M. Mokhtar, "Absolute Polar Duty Cycle Division Multiplexing: An economical and spectral efficient multiplexing technique", presented at 5th IFIP International Conference on Wireless and OpticalCommunications Networks, Surabaya, (2008).

[4]. Amin Malekmohammadi, M.K.Abdullah, A.F.Abas, G.A.Mahdiraji, M.Mokhtar, "Absolute Polar Duty Cycle Division Multiplexing (APDCDM); Technique for Wireless Communications", presented at International Conference on Computer and Communication Engineering 2008, Kuala Lumpur, Malaysia, May 13-15, 2008.

[5]. M.K.Abdullah, Amin Malekmohammadi, A.F.Abas, G.A.Mahdiraji, M.Mokhtar," $3 \times 2.5 \mathrm{~Gb} / \mathrm{s}$ Optical Absolute Polar Duty Cycle Division Multiplexing Transmission Systems".Proceedings of IEEE International Conference on Telecommunications , 26-27 August 2008 